\title{
Theoretical profiles of light alkali resonance lines for brown dwarf atmosphere conditions
}

\author{
N. F. Allard ${ }^{1,2}$, F. Allard ${ }^{3}$, and J. F. Kielkopf ${ }^{4}$ \\ ${ }^{1}$ Institut d'Astrophysique de Paris, UMR 7095, CNRS, Université Pierre et Marie Curie, 98bis boulevard Arago, 75014 Paris, \\ France \\ e-mail: allard@iap.fr \\ 2 Observatoire de Paris-Meudon, LERMA, 92195 Meudon Principal Cedex, France \\ 3 CRAL-ENS, Lyon, France \\ ${ }^{4}$ Department of Physics, University of Louisville, Louisville, KY 40292, USA
}

Received 31 March 2005 / Accepted 19 May 2005

\begin{abstract}
The analysis of the far wings of resonance lines of alkali elements in brown dwarf spectra requires their accurate determination. A unified theory of collisional line profiles has been applied for the evaluation of absorption profiles of alkalis perturbed by helium and molecular hydrogen. The study of the dependence on temperature of the far wings of $\mathrm{Li}-\mathrm{He}$ and $\mathrm{Li}-\mathrm{H}_{2}$ collisional profiles is reported.
\end{abstract}

Key words. line: profiles - stars: low-mass, brown dwarfs

\section{Introduction}

Alkali metals are the last optical opacity source to condense out to dust grains in cool substellar atmospheres. Their resonance lines provide a pseudo-continuum that shapes the emitted spectrum from the UV to the Near-Infrared spectral range. Model atmosphere, synthetic spectra and color predictions rely therefore on an adequate treatment of the far wings of alkali resonance lines in the presence of high densities of $\mathrm{H}_{2}$ and $\mathrm{He}$ perturbers.

This paper is a continuation of Allard et al. (2003) where we presented the first brown dwarf atmosphere models based on absorption profiles of sodium and potassium perturbed by $\mathrm{He}$ and $\mathrm{H}_{2}$ calculated in a unified line shape semi-classical theory (Allard et al. 1999) using molecular potentials of Pascale (1983) to describe the alkali-He interaction and those of Rossi \& Pascale (1985) for the alkali- $\mathrm{H}_{2}$ interaction. This work has been extended here to the case of lithium line profiles.

At sufficiently low densities of the perturbing gas, two different approximations are often used, depending on whether one is interested in the core of the spectral line or in its wing. In dealing with the core of the line, the impact approximation is generally used and leads to a Lorentzian profile which becomes totally unrealistic in the wing. On the other hand, the wings of the line are usually described by the one-perturber approximation, that is, the intensity distribution obtained if only one perturbing atom is interacting with the radiating atom. Although the intensity of the wings calculated with the one-perturber distribution is proportional to the pressure, the shape in this approximation is independent of pressure. This contradicts experimental observations on the satellite bands of alkali metals perturbed by rare gas (Allard \& Kielkopf 1982). As pointed out in Allard et al. (2003) the low density limit is acceptable if the density of perturbers at the depth of formation of the wings is less than $10^{19} \mathrm{~cm}^{-3}$ for $\mathrm{Na}$ and $\mathrm{K}$ perturbed by $\mathrm{He}$ or $\mathrm{H}_{2}$, but is not correct for higher densities where multiple collision effects have to be included. This cannot be taken into account in the uniform approximation of the Franck-Condon theory (Szudy \& Baylis 1975, 1996) used by Burrows \& Volobuyev (2003).

The $\mathrm{Li}-\mathrm{He} / \mathrm{H}_{2}$ profiles presented here are calculated within a unified theory which takes into account multiple collision effects (Allard et al. 1999).

After we recall the approximations used in our theoretical treatment of the collision to justify the use of a molecular representation, we focus our study on theoretical $\mathrm{Li}$ line profiles perturbed by $\mathrm{He}$ and $\mathrm{H}_{2}$ and their dependence on temperature.

\section{Theory}

A unified theory of spectral line broadening has been developed to calculate neutral atom spectra given the interaction and radiative transition moments for relevant states of the radiating atom with other atoms, the perturbers, in its environment. Complete details and the derivation of the theory are given by Allard et al. (1999). Our approach is based on the quantum theory of spectral line shapes of Baranger (1958a,b) developed in 
an adiabatic representation to include the degeneracy of atomic levels (Royer 1974, 1980; Allard et al. 1994).

\subsection{General expression for the spectrum in an adiabatic representation}

The spectrum $I(\omega)$ can be written as the Fourier transform of the dipole autocorrelation function $\Phi(s)$,

$I(\omega)=\frac{1}{\pi} \operatorname{Re} \int_{0}^{+\infty} \Phi(s) \mathrm{e}^{-\mathrm{i} \omega s} \mathrm{~d} s$.

Here,

$$
\begin{aligned}
\Phi(s) & =\operatorname{Tr} \rho \boldsymbol{D}^{\dagger} \mathrm{e}^{\frac{\mathrm{i} s \boldsymbol{H}}{\hbar}} \boldsymbol{D} \mathrm{e}^{\frac{-\mathrm{i} s \boldsymbol{H}}{\hbar}} \\
& =\left\langle\boldsymbol{D}^{\dagger}(0) \boldsymbol{D}(s)\right\rangle
\end{aligned}
$$

is the autocorrelation function of the dipole moment $\boldsymbol{D}$ in the Heisenberg representation (Allard \& Kielkopf 1982),

$\boldsymbol{D}(s) \equiv \mathrm{e}^{\frac{\mathrm{isH}}{\hbar}} \boldsymbol{D} \mathrm{e}^{\frac{-\mathrm{isH}}{\hbar}}$.

We use the notation

$$
\langle()\rangle \equiv \operatorname{Tr} \rho()
$$

where $\operatorname{Tr}$ denotes the trace operation, $\rho$ is the density matrix,

$\rho \equiv \frac{\mathrm{e}^{-\beta \boldsymbol{H}}}{\operatorname{Tr} \mathrm{e}^{-\beta \boldsymbol{H}}}$,

where $\beta$ is the inverse temperature $(1 / k T)$. We will devote the next section to the study of $\boldsymbol{H}$, the total Hamiltonian, for the specific case of alkalis perturbed by noble gas atoms or $\mathrm{H}_{2}$ molecules.

For an isolated line, we have shown (Allard et al. 1999) that the normalized line shape $J_{\alpha}(\Delta \omega)$, in the uncorrelated perturbers approximation, is given by

$$
J_{\alpha}(\Delta \omega)=\boldsymbol{F} \boldsymbol{T}\left[\mathrm{e}^{n_{p} g_{\alpha}(s)}\right]
$$

where $\alpha=(i, f)$ denotes a transition from initial state $i$ to final state $f$ and, $n_{p}$ is the perturber density. We use the semiclassical treatment for the collision problem, in which the nuclear motion is associated with a rectilinear trajectory at a single mean velocity $\bar{v}$,

$$
\bar{v}=(8 k T / \pi \mu)^{1 / 2},
$$

where $\mu$ is the reduced mass of the atoms.

We have from Allard et al. $(1994,1999)$ that $g_{\alpha}(s)$ can be written as

$$
\begin{aligned}
g_{\alpha}(s)= & \frac{1}{\sum_{e, e^{\prime}}(\alpha)\left|d_{e e^{\prime}}\right|^{2}} \sum_{e, e^{\prime}}{ }^{(\alpha)} \\
& \times \int_{0}^{+\infty} 2 \pi \rho \mathrm{d} \rho \int_{-\infty}^{+\infty} \mathrm{d} x \tilde{d}_{e e^{\prime}}[R(0)] \\
& \times\left[\mathrm{e}^{\frac{i}{\hbar} \int_{0}^{s} \mathrm{~d} t V_{e^{\prime} e}[R(t)]} \tilde{d}_{e e^{\prime}}^{*}[R(s)]-\tilde{d}_{e e^{\prime}}[R(0)]\right] .
\end{aligned}
$$

The separation of the radiator and perturber is $R(t)=\left[\rho^{2}+(x+\bar{v} t)^{2}\right]^{1 / 2}$ with $\rho$ the impact parameter of the perturber trajectory and $x$ the position of the perturber along its trajectory at time $t=0$ (Fig. 1). Since atomic states

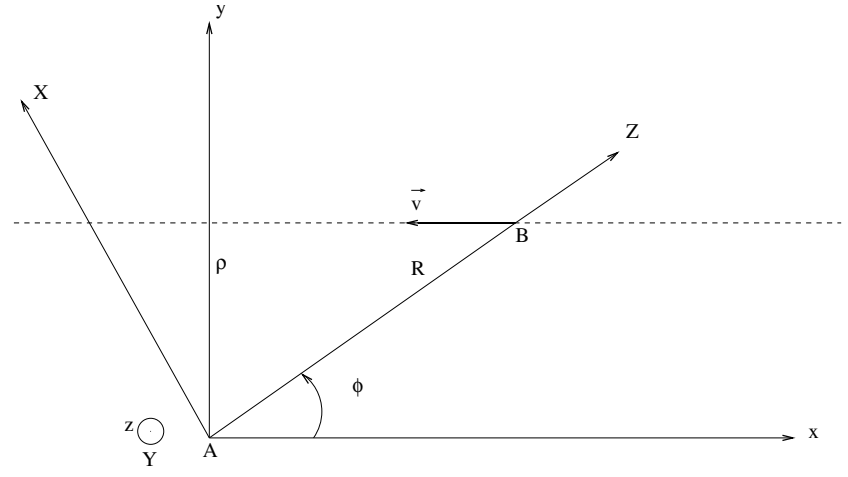

Fig. 1. The two coordinate systems. $O Z$ is the internuclear axis between the alkali and the perturber. Oxyz is the fixed frame and $\mathrm{O} X Y Z$ is the rotating frame. $Y$ and $z$ are directed out of the collision plane.

are usually degenerate, there are in general several different energy surfaces which tend to the same asymptotic energy as $R \rightarrow \infty$. The sum $\sum_{e, e^{\prime}}^{(\alpha)}$ is over all pairs $\left(e, e^{\prime}\right)$ such that $\omega_{e^{\prime}, e}(R) \rightarrow \omega_{\alpha}$ as $R \rightarrow \infty$. The transition frequency

$\omega_{e^{\prime} e}(R) \equiv\left(E_{e^{\prime}}(R)-E_{e}(R)\right) / \hbar, \quad e \in \varepsilon_{i}, e^{\prime} \in \varepsilon_{f}$

tends to the isolated radiator frequency

$\omega_{\alpha} \equiv \omega_{f i} \equiv\left(E_{f}^{\infty}-E_{i}^{\infty}\right) / \hbar$

as the perturbers get sufficiently far from the radiator:

$\omega_{e^{\prime} e}(R) \rightarrow \omega_{f i} \quad$ as $\quad R \rightarrow \infty, \quad e \in \varepsilon_{i}, e^{\prime} \in \varepsilon_{f}$.

The total line strength of the transition is $\sum_{e, e^{\prime}}{ }^{(\alpha)}\left|d_{e e^{\prime}}\right|^{2}$. The potential energy for a state $e$ is

$V_{e}[R(t)]=E_{e}[R(t)]-E_{e}^{\infty} ;$

the difference potential is

$\Delta V(R) \equiv V_{e^{\prime} e}[R(t)]=V_{e^{\prime}}[R(t)]-V_{e}[R(t)] ;$

and we defined a modulated dipole (Allard et al. 1999) with

$\tilde{d}_{e e^{\prime}}[R(t)]=d_{e e^{\prime}}[R(t)] \mathrm{e}^{-\frac{\beta}{2} V_{e}[R(t)]}$.

In the above, we neglected the influence of the potentials $V_{e}(r)$ and $V_{e^{\prime}}(r)$ on the perturber trajectories, which remain straight lines. Although we should drop the Boltzmann factor $\mathrm{e}^{-\beta V_{e}(R)}$ for consistency with our straight trajectory approximation, by keeping it we improve the result in the wings.

\subsection{Electronic Hamiltonian}

In this section we consider the resonance lines of alkalis broadened by $\mathrm{He}$ or $\mathrm{H}_{2}$ collisions. Owing to the large energy difference between the ground state and the first excited states, and the low temperatures (between 500 to $3000 \mathrm{~K}$ ) involved in the calculations of atmosphere models of extra-solar planets and brown dwarfs, the noble gas atom (or $\mathrm{H}_{2}$ molecule) is assumed to remain in its ground state, while the ground state and first excited states of the alkali are collisionally decoupled. For the transitions considered here, the lower level of the alkali atom is a ${ }^{2} S_{\frac{1}{2}}$ state. The noble gas atom is without spin it and remains 


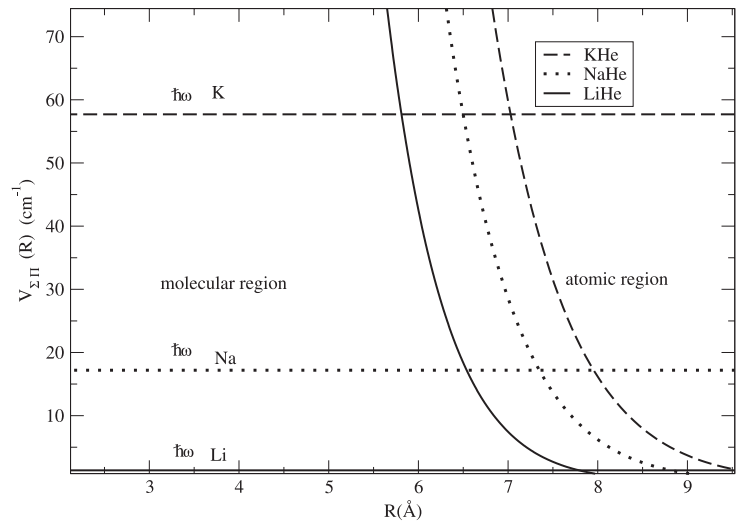

(a) $V_{\Sigma \Pi}(R)$

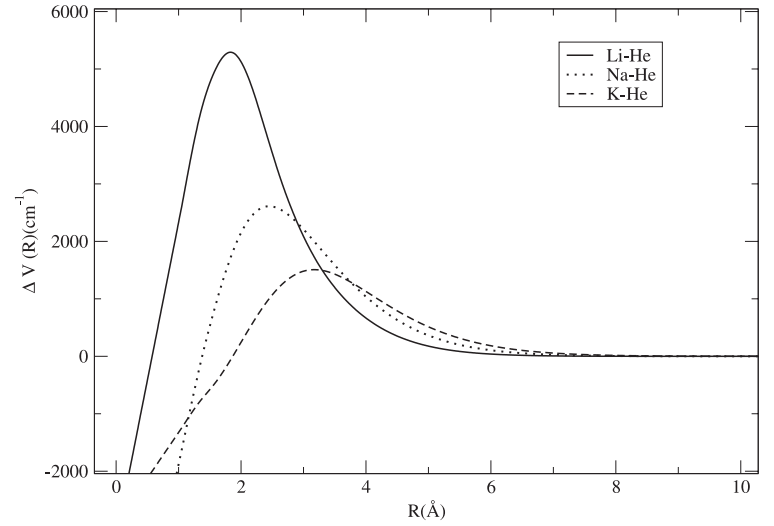

(b) $\Delta V(R)$.

Fig. 2. The fine structure splitting $\hbar \omega$ compared to the variation of $V_{\Sigma \Pi}(R)$, the splitting between the $|\Sigma\rangle$ and $|\Pi\rangle$ states, and $\Delta V(R)$ for the $A^{2} \Sigma-X^{2} \Sigma$ transition as a function of the internuclear distance $R$. The potential energies are tabulated by Pascale (1983).

in its ground ${ }^{1} \mathrm{~S}_{0}$ state. The interaction for the lower state of the system is then spherically symmetric about the alkali. We consider the system to be composed of a radiating alkali atom $A$ in collision with an atom or molecule $B$, separated from $A$ by the distance $R$. In the case of an alkali- $\mathrm{H}_{2}$ collision, $B$ is situated at the center of mass of $\mathrm{H}_{2}$.

To describe the collision, we introduce two reference frames with common origin at the center of mass of atom $A$, as illustrated in Fig. 1. A fixed-frame $\mathrm{O} x y z$ is for atomic representation with the quantization axis $O z$ perpendicular to the collision plane. A rotating frame $\mathrm{OXYZ}$ is for molecular representation, the internuclear axis being the quantization axis. The passage from the fixed to the rotating frame is described by the Euler angles $\left(\phi, \frac{\pi}{2}, \frac{\pi}{2}\right)$.

We adopt the physical model of Nikitin (1965), in which we consider an alkali atom composed of a core and an excited $p$ electron. The presence of the perturber at a distance $R$ is equivalent to an induced electric dipole at distance $R$. The problem reduces to a one-electron problem in which the radiator Hamiltonian is divided into $\boldsymbol{H}_{A}$, the electrostatic Hamiltonian, and $\boldsymbol{H}_{\mathrm{SO}}$, the spin-orbit coupling of the alkali. The total electronic Hamiltonian can be written

$\boldsymbol{H}=\boldsymbol{H}_{A}+\boldsymbol{H}_{B}+\boldsymbol{V}+\boldsymbol{H}_{\mathrm{SO}}$

$\boldsymbol{H}_{A}$ and $\boldsymbol{H}_{B}$ are the electrostatic Hamiltonians of the separated systems $A$ and $B . V$ is the electrostatic interaction of $A$ and $B$.

In the semi-classical treatment, the problem reduces to the solution of the time-dependent Schrödinger equation:

$\boldsymbol{H} \Psi(t)=i \hbar \frac{\partial \Psi(t)}{\partial t}$.

The spin-orbit Hamiltonian $\boldsymbol{H}_{\mathrm{SO}}$ is diagonal in the $|J, m\rangle$ or $|J, M\rangle$ representation, while $\boldsymbol{V}$, symmetric about the internuclear axis, is diagonal in a molecular basis $\left|\Lambda M_{L}\right\rangle$ where $\Lambda=$ $\left|M_{L}\right|$. Taking into account the symmetry in a reflection through any plane containing the internuclear axis, we may be expand $\left|\Lambda M_{L}\right\rangle$ as

$\left|\Lambda M_{L} ; \pm\right\rangle=\sum_{i} a_{i}^{\Lambda}\left(\left|L_{i} M_{L}\right\rangle( \pm)(-1)^{M_{L}}\left|L_{i}-M_{L}\right\rangle\right)$.
Although it is impossible to diagonalize the total Hamiltonian in either the atomic or the molecular basis, it is possible to use a basis which minimizes the off-diagonal elements and drop them. The system may then be described by a Hund's case (Nikitin 1965; Masnou-Seeuws \& McCarroll 1974).

\subsection{Case of alkalis with small fine structure}

We will now restrict our study to the case of the light alkalis which present a small fine structure: $\mathrm{Li}, \mathrm{Na}$, and $\mathrm{K}$.

For large $R, \boldsymbol{H}_{\mathrm{SO}} \gg \mathbf{V}$, and the orbital angular momentum $L$ of the electron is coupled with its spin $S$. The Hamiltonian is diagonal in the $|J, M\rangle$ representation. We may define the finestructure splitting $\hbar \omega$ by the relation

$\hbar \omega=\left\langle{ }^{2} \mathrm{P}_{\frac{3}{2}}\left|\boldsymbol{H}_{\mathrm{SO}}\right|^{2} \mathrm{P}_{\frac{3}{2}}\right\rangle-\left\langle{ }^{2} \mathrm{P}_{\frac{1}{2}}\left|\boldsymbol{H}_{\mathrm{SO}}\right|^{2} \mathrm{P}_{\frac{1}{2}}\right\rangle$.

Values of $\hbar \omega$ corresponding to $\mathrm{Li}, \mathrm{Na}, \mathrm{K}$, are respectively equal to $0.334,17.19$, and $57.7 \mathrm{~cm}^{-1}$. When $R$ is small, $\boldsymbol{V} \gg \boldsymbol{H}_{\mathrm{SO}}$, and $L$ is coupled with the electric field along the internuclear axis. The eigenstates are then the molecular $|\Sigma\rangle$ and $|\Pi\rangle$ states. Denoting by $V_{\Sigma \Pi}$ the difference between the eigenvalues of $\boldsymbol{V}$ for the same asymptotic excited atomic state, we have

$V_{\Sigma \Pi}=\langle\Sigma|\boldsymbol{V}| \Sigma\rangle-\langle\Pi|\boldsymbol{V}| \Pi\rangle$.

Figure 2a displays $V_{\Sigma \Pi}(R)$, the variation of the splitting between $|\Sigma\rangle$ and $|\Pi\rangle$ states as a function of the internuclear distance $R$ compared to the fine structure splitting considered to be independent of $R$. Here we show the cases of $\mathrm{Li}, \mathrm{Na}, \mathrm{K}$ perturbed by He. Similar results are obtained for these alkalis perturbed by $\mathrm{H}_{2}$.

The shape of the line wing is sensitive to $\Delta V(R)$, the difference between the ground and excited state interaction potential. When $\Delta V(R)$ for a given transition goes through an extremum, a relatively wider range of interatomic distances contribute to the same spectral frequency, resulting in an enhancement, or satellite, in the line wing (Allard 1978).

Figure $2 \mathrm{~b}$ shows the $B^{2} \Sigma-X^{2} \Sigma$ potential energy differences which exhibit a maximum and may lead to the formation of satellites on the resonance lines. The maxima in $\Delta V(R)$ in 
Table 1. Validity conditions of the Hund's cases.

\begin{tabular}{ccc}
\hline \hline Validity condition & Hund's case & Representation \\
\hline$V_{\Sigma \Pi} \gg \hbar \omega \gg \hbar \dot{\phi}$ & $a$ & $\left|\Lambda M_{L}\right\rangle\left|S M_{S}\right\rangle$ or $\left|\Lambda M_{L} ; \pm\right\rangle\left|S m_{S}, \pm\right\rangle$ \\
$V_{\Sigma \Pi} \gg \hbar \dot{\phi} \gg \hbar \omega$ & $b$ & $\left|\Lambda M_{L} ; \pm\right\rangle\left|S m_{S}\right\rangle$ \\
$\hbar \omega \gg V_{\Sigma \Pi} \gg \hbar \dot{\phi}$ & $c$ & $|J M\rangle$ or $|J M \pm\rangle$ \\
$\hbar \dot{\phi} \gg V_{\Sigma \Pi} \gg \hbar \omega$ & $d$ & $\left|L m_{L}\right\rangle\left|S m_{S} M\right\rangle$ \\
$\hbar \omega \gg \hbar \dot{\phi} \gg V_{\Sigma \Pi}$ & $e$ & \\
$\hbar \dot{\phi} \gg \hbar \omega \gg V_{\Sigma \Pi}$ & $f$ & $|J m\rangle$ \\
\hline
\end{tabular}

these cases occur at small internuclear distances $(R \sim 2-3 \AA)$. A change of coupling from an atomic representation to a molecular representation occurs around the the internuclear distance for which $V_{\Sigma \Pi} \sim \hbar \omega$. Figure 2 shows that for the cases considered here, this change of coupling takes place in the region outside where $\Delta V(\mathrm{R})$ goes through a maximum.

As the line wing intensities are most sensitive to the values of the difference potential at short internuclear distances we will use a molecular representation. In that case the axis of quantization is the rotating internuclear axis. We then introduce two possible molecular bases: $\left|\Lambda M_{L}\right\rangle\left|S M_{S}\right\rangle$ and $\left|\Lambda M_{L}\right\rangle\left|S m_{S}\right\rangle$. In this last choice the system is represented by molecular states, for which the orbital angular momentum $L$ is quantized along the molecular axis $(\mathrm{OZ})$ whereas the spin $S$ is quantized along the $(\mathrm{O} z)$ axis and remains fixed in space. However, when a rotating axis is used as the axis of quantization, off-diagonal elements in the Hamiltonian will appear due to the time dependence of the basis (Masnou-Seeuws \& McCarroll 1974). Consider, for example, the expansion of a state in terms of the basis states $\left|\Lambda M_{L}\right\rangle\left|S m_{s}\right\rangle$ in the rotating frame,

$\Psi=\sum_{\Lambda, M_{L}, S, m_{s}} b_{i}\left|\Lambda M_{L}\right\rangle\left|S m_{s}\right\rangle$.

These states have rotated an angle $\phi$ from the state at the initiation of the collision for which $t=-\infty$. The state $\Psi$ has a time derivative

$\dot{\Psi}=\sum_{\Lambda, M_{L}, S, m_{s}} \dot{b_{i}}\left|\Lambda M_{L}\right\rangle\left|S m_{s}\right\rangle-i \dot{\phi} L_{Y}\left|\Lambda M_{L}\right\rangle\left|S m_{s}\right\rangle$

and Eq. (17) becomes

$i \hbar \frac{\partial \Psi(t)}{\partial t}=\left(\boldsymbol{H}-\hbar \dot{\phi} L_{Y}\right) \Psi(t)$

The rotation of the basis states can be simulated by using an effective Hamiltonian which depends on the basis chosen. The order of magnitude of the rotational coupling is $\hbar \dot{\phi}$. Thus we have

(i) $\boldsymbol{H}^{\prime}=\boldsymbol{H}-\hbar \dot{\phi} J_{Y}$ for the $\left|\Lambda M_{L}\right\rangle\left|S M_{s}\right\rangle$ or $|J M\rangle$ basis, and

(ii) $\boldsymbol{H}^{\prime}=\boldsymbol{H}-\hbar \dot{\phi} L_{Y}$

in the $\left|\Lambda M_{L}\right\rangle\left|S m_{S}\right\rangle$ basis.

Since $\boldsymbol{H}^{\prime}$ contains operators that do not commute, the eigenfunctions of $\boldsymbol{H}^{\prime}$ have no particular symmetry property and Eq. (17) leads to a set of coupled equations. If one of the operators dominates, it is simplest to choose a representation in which this operator is diagonal. If the non-diagonal elements of $\boldsymbol{H}^{\prime}$ can be considered negligible in this representation, the system may be described by a Hund's case.

In the molecular region the system is represented either by Hund's case $a$ if the rotation is slow, or by Hund's case $b$ if the rotation is rapid. For large $R$, in the atomic region, the system may be represented by Hund's case $c$. The total angular momentum $J$ of the alkali atom may be assumed to follow the rotation of the internuclear axis. The different Hund's cases are summarized in Table 1.

Figure 3 displays the relative positions of $V_{\Sigma \Pi}, \hbar \omega$, and $\hbar \dot{\phi}$ in the case of $\mathrm{Na}-\mathrm{He}$ and $\mathrm{K}-\mathrm{He}$ collisions for temperature varying from 500 to $3000 \mathrm{~K}$. At small values of $R, V_{\Sigma \Pi} \gg \hbar \dot{\phi} \gg \hbar \omega$, and it is appropriate to use the Hund's case $b$. Figure $3 \mathrm{~b}$ shows that for $T \sim 1000 \mathrm{~K}, \hbar \dot{\phi} \sim \hbar \omega$ in the region of the maximum of the corresponding $\Delta V(R)$, and the fine structure should be included. This has been done for the calculations of the $\mathrm{K}-\mathrm{H}_{2}$ collision profile considering the fine structure as a constant. This last contribution is small compared to the maximum of $\Delta V(R)$, and its inclusion does not affect the position of the $\mathrm{K}-\mathrm{H}_{2}$ line satellite (Allard 2005). A large uncertainty is expected in the calculation of the potentials at small internuclear distance. More accurate calculations of the molecular potentials including fine structure have to be achieved for a better determination of the intensity of the wings in that region of the spectrum.

In summary, as the line wing intensities are most sensitive to the values of the difference potential at relatively short internuclear distances, we will use Hund's case $b$ to calculate the wings and Hund's case $c$ for the center of the $D_{1}$ and $D_{2}$ lines mostly sensitive to large distances. 


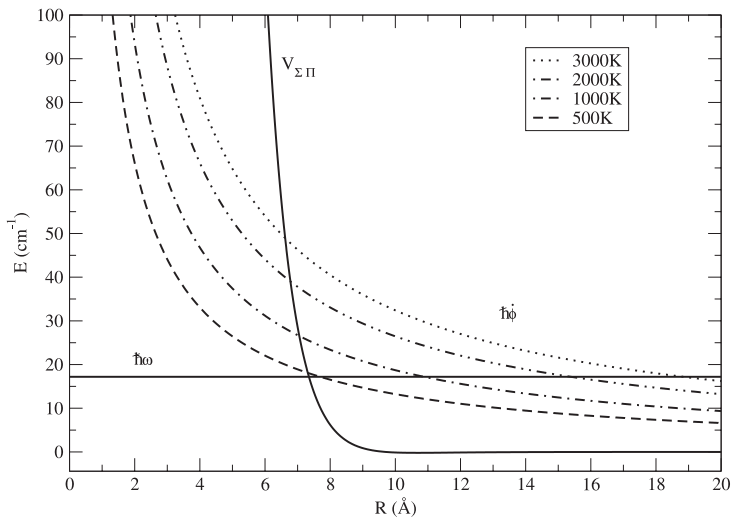

(a) $\mathrm{NaHe}$.

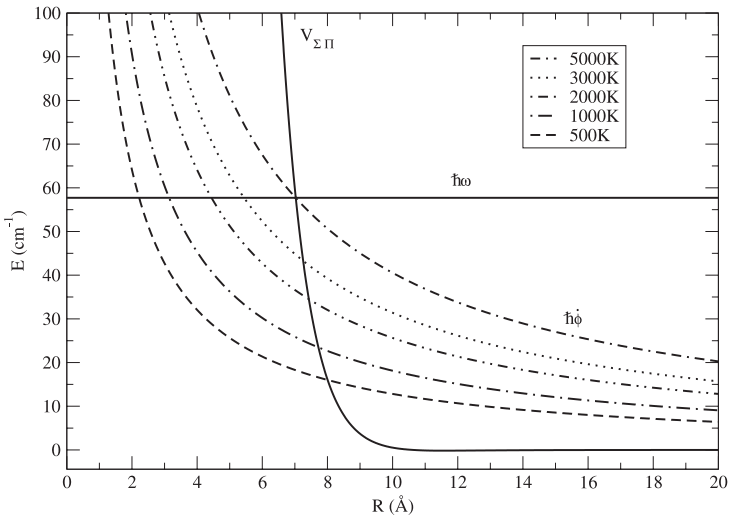

(b) $\mathrm{KHe}$.

Fig. 3. Variation of $\hbar \dot{\phi}$ and the splitting $V_{\Sigma \Pi}$ between the $|\Sigma\rangle$ and $|\Pi\rangle$ states as a function of the internuclear distance $R$ compared to the fine structure splitting $\hbar \omega$.

\section{Theoretical spectra of Li perturbed by $\mathrm{He} / \mathrm{H}_{2}$}

In this section we consider the absorption spectrum of the Li $(2 s-2 p)$ resonance transition in the presence of helium or molecular hydrogen at high density. Hund's case $b$ is valid in the molecular region because of the very small fine structure $\left(\hbar \omega=0.334 \mathrm{~cm}^{-1}\right)$. Line wing intensities are functions of the excited and ground state interaction interatomic potentials. Knowledge of the potential energies is of great importance to achieve accurate evaluations of the line profiles. Molecularstructure calculations which neglect the spin-orbit interaction have been performed by Pascale (1983) to obtain the adiabatic potentials of alkali-He systems, and by Rossi \& Pascale (1985) for alkali- $\mathrm{H}_{2}$ systems for the $C_{2 v}$ and $C_{\infty}$ symmetries. For the symmetry $C_{2 v}$ the $\mathrm{H}_{2}$ orientation is perpendicular to the $\mathrm{H}_{2}$ alkali line, whereas for the $C_{\infty}$ symmetry all atoms are colinear.

In Figs. 4 and 6 we show the energies of the $2 s-2 p$ states of the $\mathrm{LiHe}$ and $\mathrm{LiH}_{2}$ system. Figures $5 \mathrm{a}$ and 7 show that the $B-X$ difference potentials for $\mathrm{Li}-\mathrm{He}$ and $\mathrm{Li}-\mathrm{H}_{2}$ go through a maximum at small internuclear distances $(R \sim 1.5-2 \AA)$. In the theoretical spectra a maximum in $\Delta V(R)$ may lead to a satellite on the "blue" side, far from the line center. An extended red wing with no satellites can be predicted from the $\mathrm{Li}-\mathrm{He}$ and Li- $\mathrm{H}_{2} A-X$ difference potentials.

Another important factor for the presence of spectral line satellites is the variation of the electric dipole transition moment during the collision, modulated by the Boltzmann factor $\mathrm{e}^{-\beta V_{e}(R)}$. Here $V_{e}$ is the ground state potential when we consider absorption profiles, or an excited state for the calculation of a profile in emission. The modulated dipole transition moments are shown in Figs. 5a, 7a, and 7b for the light alkalis. Dipole transition moments have been provided by Pascale (2003) for $\mathrm{Li}-\mathrm{He}$. Because of the lack of knowledge of the variation of the electric dipole moment with the internuclear distance for the $\mathrm{Li}-\mathrm{H}_{2}$ system we had to assume that it remains constant all along the collision and equal to its asymptotic value, but modulated by the Boltzmann factor.

Figure 8 shows the theoretical Li line profiles perturbed by $\mathrm{He}$ and by $\mathrm{H}_{2}$, and their dependence on temperature. We note the presence of a far blue satellite about $0.5 \mu \mathrm{m}$ and $0.48 \mu \mathrm{m}$

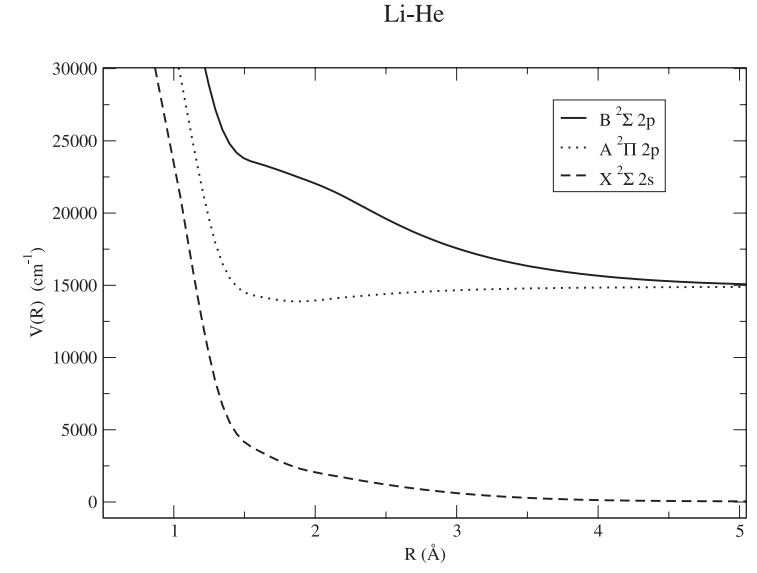

Fig. 4. Energies of the lowest states of the $\mathrm{Li}-\mathrm{He}$ system. The $B^{2} \Sigma-X^{2} \Sigma$ transition is responsible for the satellite at $0.5 \mu \mathrm{m}$, as shown in Fig. $5 b$.

in respectively the $\mathrm{Li}-\mathrm{He}$ and $\mathrm{Li}-\mathrm{H}_{2}$ resonance line wing. The appearance of the satellite is very sensitive to temperature due to the fast variation of the modulated transition dipole moment with temperature in the region of the maximum of $\Delta V(R)$ where the satellites are formed. No line satellite appears for the symmetry $C_{\infty}$ because the modulated transition dipole moment is too weak.

Emission spectra of hot dense gases in a ballistic compressor were measured by Lalos \& Hammond (1962) in a range of temperature from 3600 to $6200 \mathrm{~K}$. They report an unidentified possibly Li-He "violet" band at about $0.53 \mu \mathrm{m}$ which would agree with our calculation. The fluorescence emission spectrum of the $\mathrm{Li}(2 \mathrm{~s}-2 \mathrm{p})$ resonance transition in presence of helium at lower pressure and temperature $(670 \mathrm{~K})$ has been obtained by Scheps et al. (1975). They observed that the red wing consists of a broad plateau which extends to $0.9 \mu \mathrm{m}$. A calculation of an emission profile for this temperature is shown for comparison in Fig. 5b. In general, more of the red wing is seen in emission experiments and more of the blue wing in absorption. 


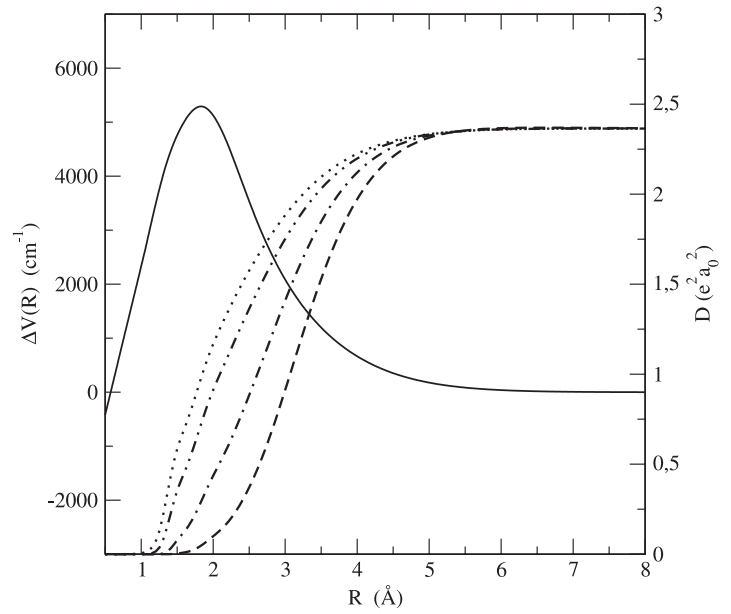

(a) Li-He modulated dipole, and the $B^{2} \Sigma-X^{2} \Sigma$ difference potential.

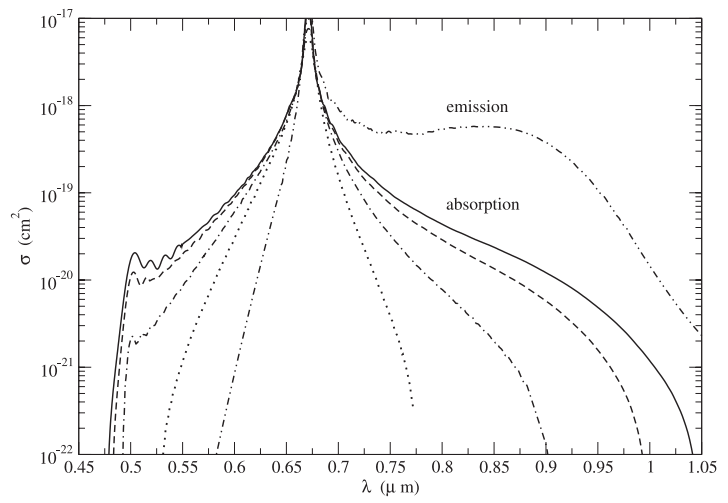

(b) Li-He profile for a density of perturbers $n_{\mathrm{He}}=10^{19}$ $\mathrm{cm}^{-3}$.

Fig. 5. Variation with temperatures of the Li-He modulated dipole and of the corresponding Li-He absorption profiles at 3000, 2000, 1000 and $500 \mathrm{~K}$ (top to bottom), an emission profile at $670 \mathrm{~K}$ is plotted for comparison.

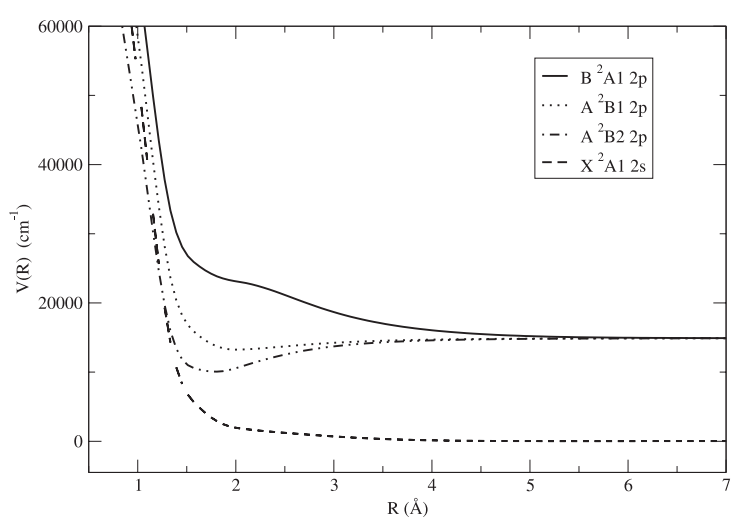

(a) Symmetry $C_{2 v}$.

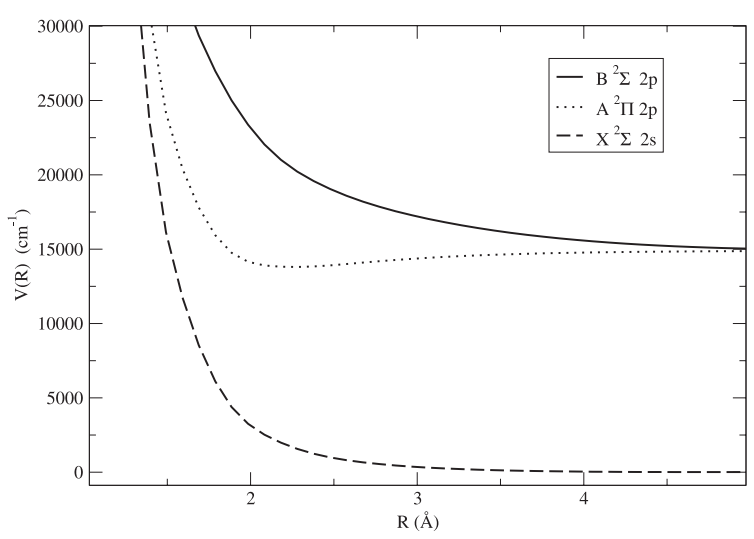

(b) Symmetry $C_{\infty}$.

Fig. 6. Energies of the $2 \mathrm{~s}-2 \mathrm{p}$ states of the $\mathrm{LiH}_{2}$ system.

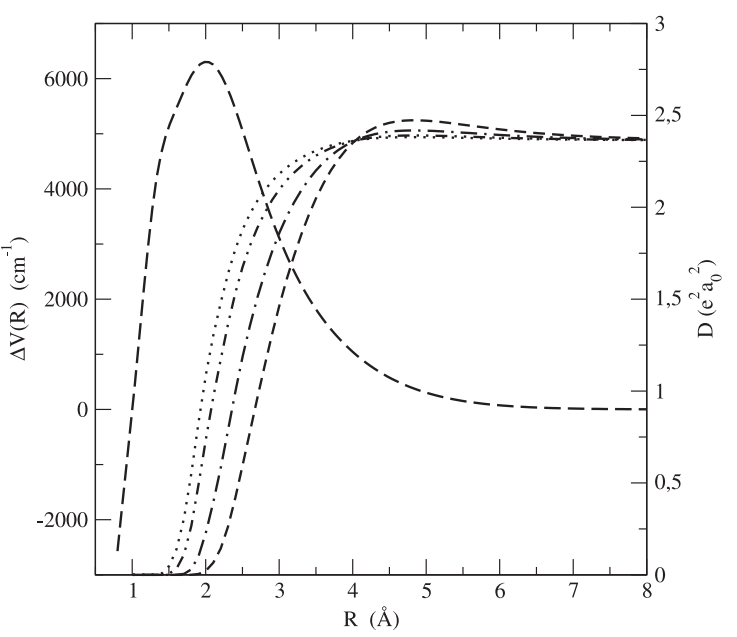

(a) Symmetry $C_{2 v}$.

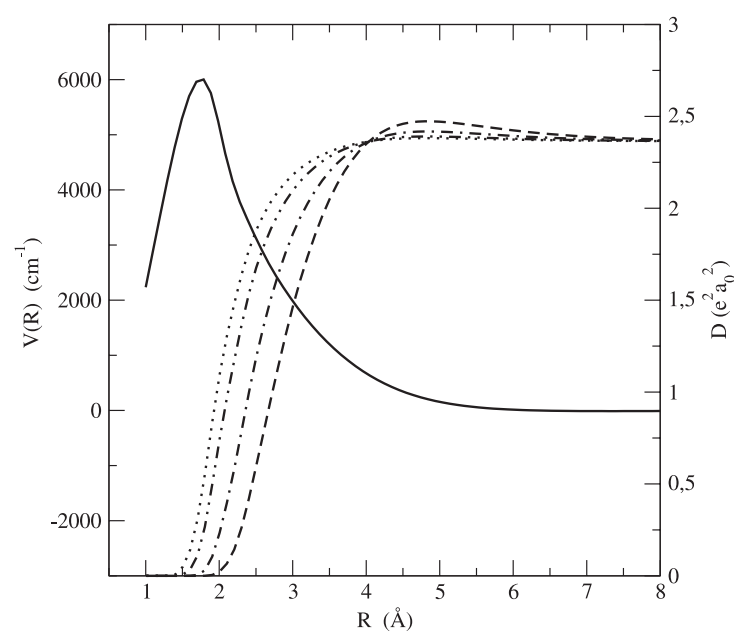

(b) Symmetry $C_{\infty}$.

Fig. 7. Variation with temperatures of the $\mathrm{Li}-\mathrm{H}_{2}$ modulated dipole at $3000,2000,1000$ and $500 \mathrm{~K}$ (top to bottom), and the difference $B^{2} \Sigma-X^{2} \Sigma$ of upper and lower state potentials for the resonance lines. 


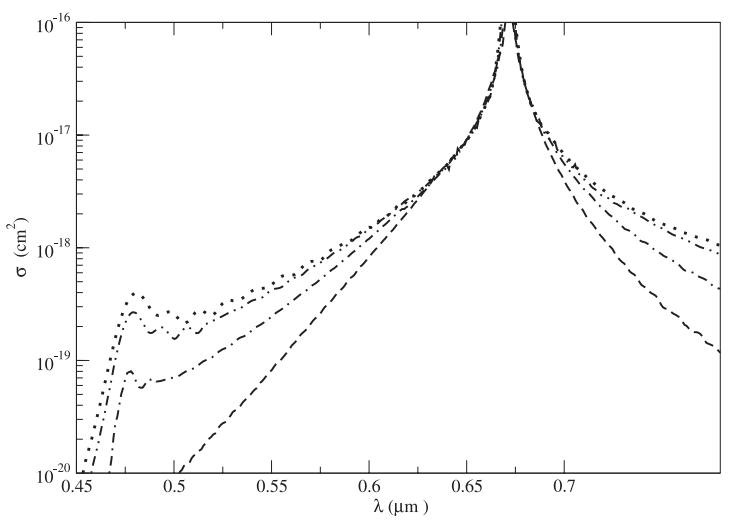

(a) Symmetry $C_{2 v}$.

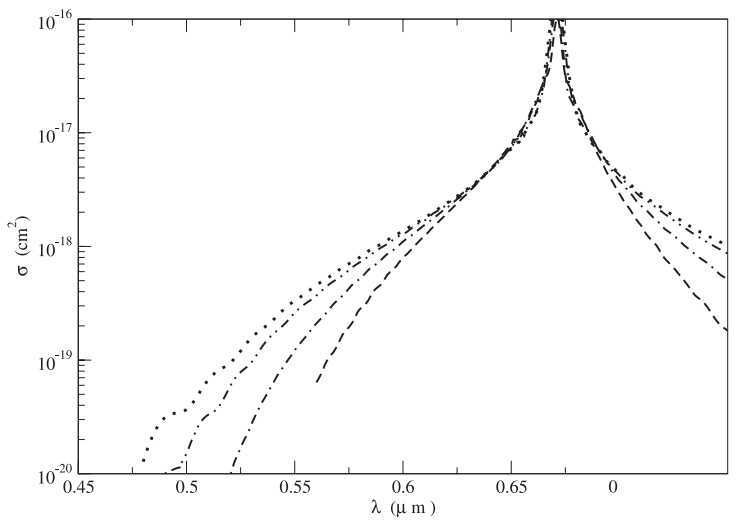

(b) Symmetry $C_{\infty}$.

Fig. 8. Variation of the line profiles of Li perturbed by $\mathrm{H}_{2}$ with temperature. The density of perturbers is $n_{\mathrm{H}_{2}}=10^{20} \mathrm{~cm}^{-3}$. The temperatures (top to bottom) are 3000, 2000, 1000 and $500 \mathrm{~K}$.

\section{Conclusions}

Our calculations of unified theory line profiles using molecular potentials to describe the interaction of alkalis perturbed by $\mathrm{He}$ and $\mathrm{H}_{2}$ have been shown to improve the comparison of synthetic spectra of brown dwarfs to observations. Nevertheless more accurate determinations of the potentials at short internuclear distance are necessary to rely on the theoretical position of the line satellites. No experimental data are presently available for comparison. The dependence of the position of the line satellite on orientation angle requires us also to know the potentials for more possible orientations of the $\mathrm{H}_{2}$ molecule. The fine structure also needs to be included to consider the cases of heavier alkalis like rubidium and cesium in order to have a fully consistent set of alkali spectral line profiles.

Acknowledgements. N.F.A. would like to thank Francoise Masnou for helpful discussions.

\section{References}

Allard, N. F. 1978, J. Phys. B., 11, 1383

Allard, N. F. 2005, in preparation
Allard, N. F., \& Kielkopf, J. F. 1982, Rev. Mod. Phys., 54, 1103

Allard, N. F., Royer, A., Kielkopf, J. F., \& Feautrier, N. 1999, Phys. Rev. A, 60, 1021

Allard, N. F., Allard, F., Hauschildt, P. H., Kielkopf, J. F., \& Machin, L. 2003, A\&A, 411, 473

Baranger, M. 1958a, Phys. Rev., 111, 481

Baranger, M. 1958b, Phys. Rev., 111, 494

Burrows, A., \& Volobuyev, M. 2003, ApJ, 583, 985

Lalos, G. T., \& Hammond, G. L. 1962, ApJ, 135, 616

Masnou-Seeuws, F., \& McCarroll, R. 1974, J. Phys. B: Atom. Molec. Phys., 7, 16, 2230

Nikitin, E. E. 1965, J. Chem. Phys., 45, 2671

Pascale, J. 1983, Phys. Rev. A, 28, 632

Pascale, J. 2003, Private communication

Rossi, F., \& Pascale, J. 1985, Phys. Rev. A, 32, 2657

Royer, A. 1974, Can. J. Phys., 52, 1816

Royer, A. 1980, Phys. Rev. A, 22, 1625

Scheps, R., Ottinger, Ch., York, G., \& Gallagher, A. 1975, J. Chem. Phys., 63, 2581

Szudy, J., \& Baylis, W. 1975, J. Quant. Spectrosc. Radiat. Transfer, 15,641

Szudy, J., \& Baylis, W. 1996, Phys. Rep., 266, 127 